Sharif University of Technology
Scientia Iranica
Transactions E: Industrial Engineering
wCIENTIA

\title{
A new proactive-reactive approach to hedge against uncertain processing times and unexpected machine failures in the two-machine flow shop scheduling problems
}

\author{
D. Rahmani* \\ Department of Industrial Engineering, K. N. Toosi University of Technology, Tehran, Iran. \\ Received 20 October 2015; received in revised form 21 February 2016; accepted 19 July 2016
}

\author{
KEYWORDS \\ Disruption; \\ Robustness; \\ Stability; \\ Nervousness; \\ Flow shop; \\ Proactive-reactive \\ approach.
}

\begin{abstract}
In this paper, a proactive-reactive approach has been considered for achieving stable and robust schedules despite uncertain processing times and unexpected machine failures in a two-machine flow shop system. In the literature, Surrogate Measures (SMs) have been developed for achieving stable and robust solutions against the occurrence of stochastic disruptions. These measures proactively provide an approximation of the real conditions of the system in the event of a disruption. Because of the discrepancies of these measures with their real values, a different approach is developed in this paper in a two-step structure. First, an initial robust schedule is produced and then, based on a multi-component measure, an appropriate reaction is adopted against unexpected machine failures. Computational results indicate that this method produces better solutions compared to the other two classical scheduling approaches considering their effectiveness and performance.

(C) 2017 Sharif University of Technology. All rights reserved.
\end{abstract}

\section{Introduction}

In the classical scheduling problems, it is assumed that the information about all jobs and their characterizations is known initially, and the objective is usually to optimize some classical performance measures such as tardiness and makespan under deterministic assumptions [1]. However, in dynamic manufacturing environments, the scheduling problems with uncertainty are addressed. In stochastic scheduling problems, the solving approaches often try to optimize the expected value or some other probabilistic measure of the objective function [2]. In uncertain scheduling problems, other measures are proposed to incorporate the uncertainty [3]. Ouelhadj and Petrovic [4] presented a survey

*. Tel.: +982184063357; Fax: +982188674858

E-mail address: drahmani@kntu.ac.ir on dynamic scheduling in manufacturing systems. In fact, because of random disruptions that may occur in the system, additional measures (such as robustness and stability) should be considered. Some disruptions that may occur in real-world manufacturing systems include:

- Machine breakdowns;

- Cancellation of orders;

- Changes in delivery times;

- Uncertain due dates;

- Uncertain processing times;

- Equipment overhaul;

- Addition or removal of operations.

In practice, the scheduling process starts by determining an initial schedule. Then, when a disruption 
arises, the initial schedule should be revised in order to maintain its feasibility and quality. The type of schedule that is actually carried out in shops is known as real schedule. Obviously, real schedule can be different from the initial schedule because of occurrence of unexpected events. This depends on the level of failures, disruptions, and the changes of the setting. In the literature, there are two strategies for obtaining high system performance from the real schedule after any disruption. These strategies are called reactive scheduling and proactive scheduling.

The reactive scheduling approach does not initially consider uncertainty, but revises and improves the schedule when unpredicted events occur. In fact, reactive scheduling is a method that provides good reactions when encountering failure and disruption. This reaction can modify the initial schedule or generate a completely new schedule. However, proactive scheduling strategy considers future disruptions when generating an initial schedule. It actually seeks a schedule that also controls the effects of future disruptions by some predictive performance measures such as robustness and stability. Optimization of stability is concerned with the deviation of the real schedule from the initial schedule. Optimization of robustness is concerned with deference in terms of objective function between initial and modified schedules [5]. Of course, a combined proactive-reactive approach can also be considered [6].

In this paper, a two-stage proactive-reactive method is presented for coping with uncertain and unexpected events. In the first stage, it is attempted to produce an initially robust schedule by using the robust optimization approach. The initial robust schedule counters the fluctuations of processing times. In the second stage, when an unexpected disruption occurs (i.e., machine failure), an appropriate reaction is adapted to rescheduling.

This paper is organized in the following manner. In Section 2, the related technical literature is reviewed and a brief description of robust optimization approach is presented. The main two-step approach of the paper is presented in Section 3. In the first step, a robust model of two-machine flow shop scheduling problem has been presented and solved, and in the second step, the appropriate reactive approach has been described. Computational results and relevant comparisons have been presented in Section 4. Finally, conclusions and recommendations for future studies have been presented in the last section.

\section{Literature review}

Flow shop is one of the most practical and realworld production environments, especially in assembly facilities [7]. Some researchers including Fattahi et al. [8] proposed mathematical models to formulate the flow shop environment and some heuristics to solve the problem.

In order to decrease the effect of uncertain processing times, some researchers considered a specific distribution function for them and solved the problem based on the stochastic optimization approach. Some other researchers used the robust optimization approach so that this approach could improve the performance of the presented schedule by facing the fluctuations of uncertain processing times concerning all possible future scenarios. Proactive scheduling methods that deal with the future fluctuations of uncertain parameters using the robust optimization approach are presented in [9-12].

Considering disruptions and unexpected events in scheduling systems, the researchers either used iteration based simulation methods [13] or attempted to develop robust and stable schedules to face these disruptions. $\mathrm{Wu}$ et al. [14] considered increase in stability of the single-machine rescheduling problem with machine breakdowns. They rescheduled the jobs in response to machine failures so that the minimum makespan could achieve a high scheduling stability. Leon et al. [15] studied the issue of robustness in the job shop environment. They assumed that the times of failure and repairing were known values, and makespan was the shop performance measure that should be minimized. For analysis of the effects of machine failures and changes of the processing times, the authors proposed a slack time based robustness measure. The most promising robustness measure is expressed as:

$$
Z_{r=1}(s)=M S_{\min }-R D 3(s)
$$

where $M S_{\min }$ is the makespan of schedule $s$ and $R D 3(s)$ is the average slack in schedule $s$. Lawrence and Sevelle [16] studied the performance of the simple dispatching heuristics against the algorithmic solution techniques in a job shop environment with uncertain processing times. A similar study was undertaken by Sabunkuglu and Karabuk [17] in which they showed that the dispatching rules for interruptions were more robust than the optimum search algorithms for offline schedules. Jensen [18] generated robust schedules in a job shop environment with respect to machine breakdowns for minimizing makespan. Jensen's idea was based on the principle that the robust optimal solution was found in the wider regions of the distribution (objective) function, while the non-robust and fragile optimal solutions were located on the narrow peaks of the distribution function. In fact, he considered the neighborhood-based robustness measure for including the schedule $s$ and all the achievable schedules. His formula is as follows: 


$$
Z_{M S_{n i b}}(s)=\frac{1}{\left|N_{1}(s)\right|} \sum_{s^{\prime} \in N_{1}(s)} M S_{\min }\left(s^{\prime}\right)
$$

where $M S_{\min }\left(s^{\prime}\right)$ is the makespan of schedule $s^{\prime}$. The neighborhood $N_{1}(s)$ contains $s$ and all feasible schedules that can be created from $s$ by interchange of two consecutive operations on the same machine. Goren and Sabuncuoglu [19] investigated the problem of robust and stable schedule with random failures in a single-machine environment. They presented two surrogate measures for robustness and stability, and used the tabu search algorithm to solve the problem. Sotskov et al. [20] presented a number of approaches based on interval processing times for the evaluation of robustness and stability in a single-machine environment. Bouyahia et al. [21] proposed a probable comprehensive approach for the robustness design of pre-scheduling, which assumed that the number of jobs to be processed on parallel machines was a random variable. They studied the total weighted flow time as an objective function. Ghezail et al. [22] proposed a qualitative graphical approach for responding to the disruptions in the flow shop problem. They proposed a graphical approach that helped the decision maker to observe the consequences of random failures and to choose the best sequence. Al-Hinai and ElMekkawy [23] produced proactive robust and stable solutions for the flexible job shop scheduling problem with random failures. They introduced a new methodology that combined the approach of insertion of non-idle time and a hybrid genetic algorithm proposed in [24].

Based on the literature, the researchers considered stability and robustness separately to face the stochastic disruptions in scheduling problems. To produce robust and stable solutions, the true value of uncertain parameters should be determined. However, since the exact values of these parameters are not specified from the start, either iteration based timeconsuming simulation methods or surrogate measures are used in the literature to obtain robust and stable solutions. Because of the discrepancies between these measures and their true values, they may not show the true performance of the system. We proposed a new proactive-reactive approach instead of SMs to overcome their weaknesses and achieve good-quality solutions. We also considered a new practical measure called "nervousness" in the two-machine flow shop scheduling in addition to the stability and robustness. Accordingly, a multi-criteria measure was presented in the reactive stage of the proposed method. In the following, the structure of the robust optimization approach used to formulate our considered problem is explained [25].

\section{Statement of contribution}

- An uncertain two-machine flow shop problem is considered;
- A two-stage method is presented to reduce the effects of uncertainty and disruption;

- A robust model is developed to create an initial robust schedule to overcome the uncertain processing times;

- A new measure called "NERS value" is proposed to apply a good reaction after a machine failure;

- Four critical factors are included in the proposed measure to select the best method for rescheduling after a disruption;

- Multiple methods to evaluate the performance of the proposed method are considered.

Robust optimization approach: The aim of the robust optimization approach is to get a set of solutions for the problem that remains robust despite the changes that may occur in the real values of data and input parameters (shown by a set of scenarios). The structure of robust optimization is briefly explained in the following section. Consider the following linear model:

$$
\min c^{T} x+d^{T} y
$$

subject to:

$$
\begin{aligned}
& A x=b, \\
& B x+C y=e, \\
& x, y \geq 0,
\end{aligned}
$$

where $x$ is the vector of decision variables and $y$ is the vector of control variables. $B$ and $C$ are coefficient vectors and $e$ is the right hand side vector. Assume that there exists a set of scenarios, $\Omega=\{1,2, \ldots, \tilde{\lambda}\}$. Under each scenario, $\lambda$, the uncertain coefficients are defined as $\left\{d^{\lambda}, B^{\lambda}, C^{\lambda}, e^{\lambda}\right\}$ and the probability of occurrence of each scenario is $p^{\lambda}\left(\sum_{\lambda} p^{\lambda}=1\right)$. Each scenario comprises a set of data that may occur in the future. Since $y$ (vector of control variables) is determined in the constraint depending on the scenario that occurs, it is defined as $y^{\lambda}$. Due to the existence of uncertain parameters, the model may become infeasible for some scenarios; in this case, $\delta^{\lambda}$ is the degree of infeasibility of the model under scenario $\lambda$. If the model is feasible, then $\delta^{\lambda}=0$. Thus, $\delta^{\lambda}$ is an error vector that will measure the infeasibility allowed in the control constraints under scenario $\lambda$. The ultimate goal of this approach is the optimization of the problem with two kinds of robustness: solution robustness, which guarantees a near-optimum solution for all the scenarios, and model robustness, which guarantees the problem solution to be feasible for all the possible scenarios. Therefore, the robust model is made as follows:

$$
\operatorname{Min} \sigma\left(x, y^{1}, y^{2}, \ldots, y^{\tilde{\lambda}}\right)+\omega \rho\left(\delta^{1}, \delta^{2}, \ldots, \delta^{\tilde{\lambda}}\right),
$$


subject to:

$$
\begin{aligned}
& A x=b, \\
& B^{\lambda} x+C^{\lambda} y+\delta^{\lambda}=e^{\lambda} \quad \forall \lambda \in \Omega, \\
& x \geq 0, \quad y^{\lambda} \geq 0, \quad \delta^{\lambda} \geq 0 \quad \forall \lambda \in \Omega .
\end{aligned}
$$

In Constrain $(6), \sigma($.$) denotes the solution robustness$ and $\rho($.$) is the model robustness. In fact, \rho($.$) is a$ penalty function for the solution possibility, which is used to penalize the deviations of control constraints under some of the scenarios. Also, coefficient $\omega$ establishes the equilibrium between the solution robustness and model robustness. Constraint (7) is the structural constraint and there is no noise. Constraint (8) is the control constraint that contains noisy coefficients. Constraint (9) ensures non-negative values for variables.

The first term in the objective function can be defined with a random variable such as $\xi=c^{T} x+d^{T} y$ that takes the value $\xi^{\lambda}=c^{T} x+d^{\lambda^{T}} y^{\lambda}$ with probability $p^{\lambda}$ under scenario $\lambda$. To define $\sigma($.$) , Mulvey et al. [11]$ used the following relation:

$$
\sigma(.)=\sum_{\lambda \notin \Omega} p^{\lambda} \xi^{\lambda}+\gamma \sum_{\lambda \in \Omega} p^{\lambda}\left(\xi^{\lambda}-\sum_{\lambda^{\prime} \in \Omega} p^{\lambda^{\prime}} \xi^{\lambda^{\prime}}\right)^{2},
$$

in which the solution has a lower sensitivity to the changes of uncertain data as $\gamma$ increases. However, since this term is quadratic, its solving will be complicated; due to this, $\mathrm{Yu}$ and li [26] defined another term for $\sigma($.$) as:$

$$
\sigma(.)=\sum_{\lambda \notin \Omega} p^{\lambda} \xi^{\lambda}+\gamma \sum_{\lambda \in \Omega} p^{\lambda}\left|\xi^{\lambda}-\sum_{\lambda^{\prime} \in \Omega} p^{\lambda^{\prime}} \xi^{\lambda^{\prime}}\right| .
$$

But since this term is non-linear, by defining a nonnegative deviational variable, the problem is converted to a linear model as follows:

$$
\begin{aligned}
\sigma(.) & =\sum_{\lambda \notin \Omega} p^{\lambda} \xi^{\lambda}+\gamma \sum_{\lambda \in \Omega} p^{\lambda}\left[\left(\xi^{\lambda}-\sum_{\lambda^{\prime} \in \Omega} p^{\lambda^{\prime}} \xi^{\lambda^{\prime}}\right)\right. \\
& \left.+2 \theta^{\lambda}\right]
\end{aligned}
$$

subject to:

$$
\begin{aligned}
& \xi^{\lambda}-\sum_{\lambda^{\prime} \in \Omega} p^{\lambda^{\prime}} \xi^{\lambda^{\prime}}+\theta^{\lambda} \geq 0, \quad \forall \lambda \in \Omega, \\
& \theta^{\lambda} \geq 0, \quad \forall \lambda \in \Omega .
\end{aligned}
$$

\section{Proposed method}

In this paper, a two-machine flow shop problem with uncertain job processing times is considered. There is some information about uncertain processing times, and they are estimated by scenarios. In addition to uncertain processing times, unexpected failures may occur in the future; but there is no predictive information in this regard. Therefore, in the first stage of the proposed method, unexpected machine failure is not considered, because they are completely unexpected events and there is no information to formulate them. Hence, in the first stage, an initial solution for scheduling is obtained by only considering the problem with uncertain processing times. The robust optimization approach is used in order for this initial solution to be a robust solution as well. Actually, to reduce the effect of uncertainty on processing times, which is a random disruption in the future, the problem is formulated using the robust optimization approach, firstly, in an attempt to produce robust initial solutions. After an initially robust schedule is determined, the machines start to process the jobs according to this schedule. However, each machine may break down during the processing of jobs. Therefore, in the second stage, a reactive approach is presented to deal with such unexpected failures. In fact, when a machine failure occurs, an appropriate reaction should be adopted to handle this disruption. Suppose that different reactions, like regeneration, right shifting, or any other heuristic reaction can be implemented following the failure. When adopting a reactive response, a multi-component measure is defined based on a classic objective and three other performance measures. This measure helps us choose the most appropriate reaction to counter the effect of machine failures. A flowchart of our proposed approach is presented in Figure 1.

\subsection{Proactive scheduling stage}

In this stage, only the problem with uncertain processing times is considered. The robust optimization approach is used to formulate the two-machine flow shop problem to reduce the effects of fluctuations of the processing times in the future. Consider a set $J=\{1,2, \ldots, n\}$ of $n$ independent jobs that require processing on each of the two machines. Assume that there exists a limited set of $\{1,2, \ldots, \tilde{\lambda}\}$. A robust solution is proactively generated as an initial schedule.

\subsubsection{Notations}

Indices:

$n \quad$ The number of jobs

$\lambda, \lambda^{\prime} \quad$ Indices for scenarios

$r \quad$ Index for positions $\{1,2, \ldots, n\}$

$j \quad$ Index for jobs $\{1,2, \ldots, n\}$

$i \quad$ Index for machines $\{1,2\}$ 


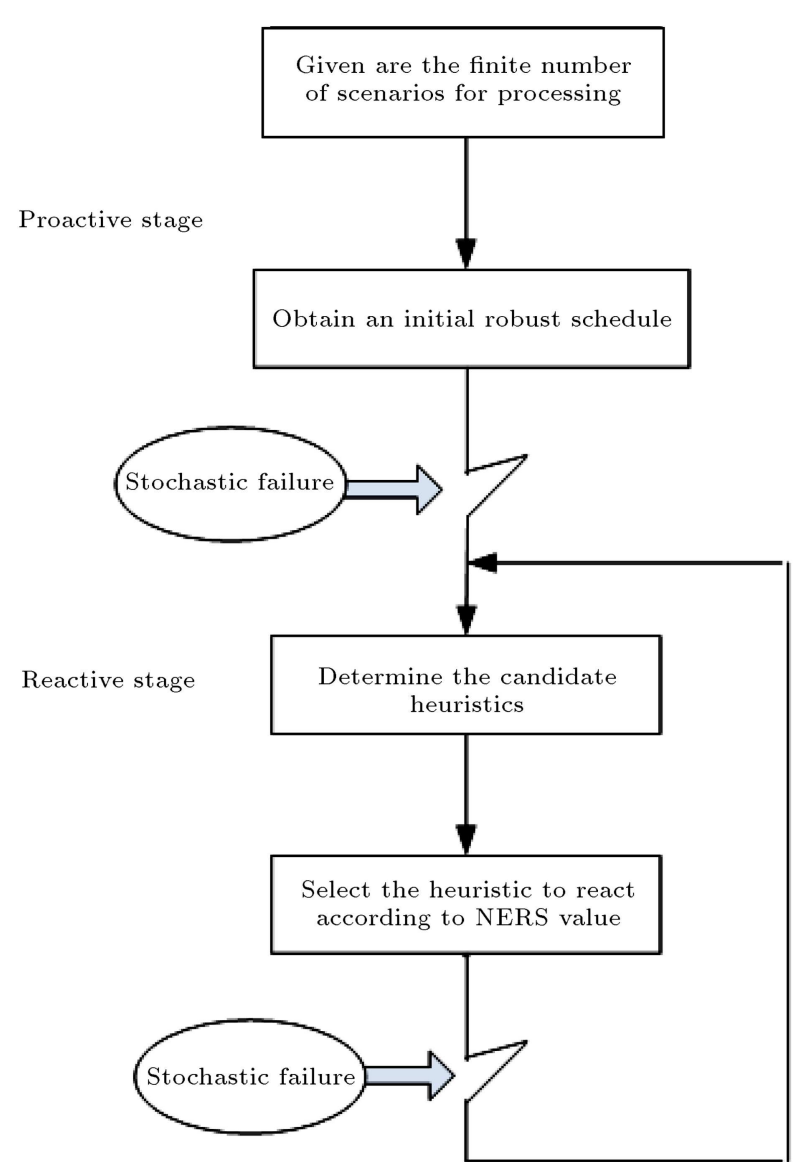

Figure 1. The flowchart of the proposed proactive-reactive method.

\section{Parameters:}

$t_{i j}^{\lambda} \quad$ The processing time of job $j$ on machine $i$ under scenario $\lambda$

$p^{\lambda} \quad$ The probability for occurrence of

scenario $\lambda$

$M \quad$ A large number

Variables:

$x_{i j r} \quad 1$ if the job $j$ is processed on machine $i$ in the position $r$; 0 otherwise

$C P_{i j r}^{\lambda} \quad$ The predictive completed time of job $j$ on the machine $i$ in the position $r$ under scenario $\lambda$

\subsubsection{Proactive robust model}

Now, the considered problem is formulated based on the robust optimization approach described in the previous section. The noteworthy point is that in this model, due to the unequal form of the existing constraints, it is not necessary to define parameter $\delta^{\lambda}$ and function $\rho\left(\delta^{1}, \delta^{2}, \ldots, \delta^{\tilde{\lambda}}\right)$ to guarantee solution robustness. Therefore, the issue of creating a balance between solution robustness and model robustness no longer exists. Therefore, our developed robust model is as follows:

$$
\begin{aligned}
\operatorname{Min} & \sum_{\lambda \in \Omega} p^{\lambda} \sum_{j=1}^{n} C P_{2 j n}^{\lambda}+\sum_{\lambda \in \Omega} p^{\lambda} \mid \sum_{j=1}^{n} C P_{2 j n}^{\lambda} \\
& -\sum_{\lambda^{\prime} \in \Omega} p^{\lambda^{\prime}} \sum_{j=1}^{n} C P_{2 j n}^{\lambda^{\prime}} \mid
\end{aligned}
$$

subject to:

$$
\begin{aligned}
& \sum_{r=1}^{n} x_{i j r}=1, \quad \forall i, \forall j, \\
& \sum_{j=1}^{n} x_{i j r}=1, \quad \forall i, \forall r, \\
& \sum_{j=1}^{n} C P_{i j(r+1)}^{\lambda} \geq \sum_{j=1}^{n} C P_{i j r}^{\lambda}+\sum_{j=1}^{n} x_{i j(r+1)} \cdot t_{i j}^{\lambda} \\
& \forall i, \forall \lambda, r=1, \ldots, n-1, \\
& \sum_{r=1}^{n} C P_{(i+1) j r}^{\lambda} \geq \sum_{r=1}^{n} C P_{i j r}^{\lambda}+\sum_{r=1}^{n} x_{(i+1) j r} \cdot t_{(i+1)}^{\lambda} \\
& \forall j, \forall \lambda, i=1, \ldots, m-1, \\
& C P_{i j r}^{\lambda} \leq M . x_{i j r} ; \quad \forall i, \forall j, \forall r, \forall \lambda \\
& x_{i j r}=\{0,1\} ; \quad \forall i, \forall j, \forall r .
\end{aligned}
$$

To linearize the objective function, a method is used and the defined parameter $\theta^{\lambda}$ has been described in the previous section [26]. Therefore, we have:

$$
\begin{aligned}
& \operatorname{Min} \sum_{\lambda \in \Omega} p^{\lambda} \sum_{j=1}^{n} C P_{2 j n}^{\lambda}+\sum_{\lambda \in \Omega} p^{\lambda}\left[\left(\sum_{j=1}^{n} C P_{2 j n}^{\lambda}\right.\right. \\
& \left.\left.-\sum_{\lambda^{\prime} \in \Omega} p^{\lambda^{\prime}} \sum_{j=1}^{n} C P_{2 j n}^{\lambda^{\prime}}\right)+2 \theta^{\lambda}\right], \\
& -\theta^{\lambda}-\sum_{j=1}^{n} C P_{2 j n}^{\lambda}+\sum_{\lambda^{\prime} \in \Omega} p^{\lambda^{\prime}} \sum_{j=1}^{n} C P_{2 j n}^{\lambda^{\prime}} \leq 0 ; \quad \forall \lambda .
\end{aligned}
$$

Thus, Objective (21) and Constraints (22) ensure that the optimal schedule conforms to the definition of robust schedule based on a linear model. Constraints (15) to (20) are necessary scenario-based constraints in a two-machine flow shop system to calculate appropriate makespan. These constraints guarantee that the robust schedule is feasible.

\subsection{Reactive scheduling stage}

Assume that in the first stage, an initial robust schedule is determined. The machines may break down during 
the processing of jobs unexpectedly. Suppose that machine $i$ fails at time $t_{f}$. In this case, the main point is to choose an appropriate heuristic as a good reaction after this failure. The important point that should be mentioned is that the proposed approach to respond to the machine failure is a reactive one. Therefore, there is no need to know the distribution function of the failure occurrence time and repair duration.

Suppose that a set of heuristic methods, $\Pi=$ $\left\{H_{1}, H_{2}, \ldots, H_{h}\right\}$, exists, which can be used after the occurrence of failure. These heuristic methods can be the right shifting, regeneration, or any other heuristic. In this research, a multicomponent measure is defined to choose the most appropriate heuristic method from the set $\Pi$. This measure is defined based on a classical objective and three other performance measures. The considered classical objective in this research is makespan that indicates scheduling effectiveness. Other performance measures include robustness, stability, and nervousness that control the unexpected disruptions. This measure is called "NERS value" (including Nervousness, Effectiveness, Robustness, and Stability). These components are described in the following subsections.

\subsubsection{Scheduling effectiveness}

This measure indicates the degree of optimality of a schedule. In this paper, this criterion is measured by the classical objective "makespan". It should be mentioned that because of disruptions such as machine failures in the system, the real completion time of affected jobs may change. The stochastic variable $C R_{i j r}$ is defined as the real completion time of job $j$ on machine $i$ at position $r$. Therefore, in a two-machine flow shop system, $\sum_{j} C R_{2 j n}$ is the real makespan for the realized schedule that explains scheduling effectiveness.

\subsubsection{Robustness}

The robustness of a schedule refers to its ability to perform well under different operational environments. In fact, robustness is concerned with the difference in objective function values before and after a disruption. It refers to the insensitivity of scheduling performance to the disruptions. Some robustness measures are based on the actual performance of the realized schedules, and some are based on regret. [3]. In this research, this measure is defined as the closeness of the realized schedule performance to the initial schedule.

It should be mentioned that in the first stage, in order to determine the initial schedule, the processing times were estimated as scenarios. In fact, some issues such as the condition of machines, the state of operators, the environmental conditions, etc. affect processing times and the real states of these issues would be determined only at schedule execution time.
A finite number of scenarios (three scenarios) were considered to define the uncertain processing times to obtain a robust initial schedule. As mentioned earlier, in robust optimization approach, one of these scenarios would occur in future. During the planning of an initial schedule, the real scenario that will really occur in the system is not determined. But, when the production process begins, before a disruption, one or more jobs have been processed on machines, and so we can find out which scenario has occurred. In fact, the compatible scenario with these completed jobs has been determined. Considering this notion, suppose $\lambda^{\prime \prime} \in \Omega$ is the scenario that has really occurred after the start of jobs processing. In this case, the real completion times without considering unexpected failures are specified based on the scenario $\lambda^{\prime \prime} \in \Omega$. Based on this matter and the definition of robustness (absolute deviation in system performance), the robustness measure is calculated as follows:

$$
\text { Robustness measure : }\left|\sum_{j} C R_{2 j n}-\sum_{j} C P_{2 j n}^{\lambda^{\prime \prime}}\right| \text {, }
$$

$\sum_{j} C R_{2 j n}$ is the real makespan of the perturbed schedule and $\sum_{j} C P_{2 j n}^{\lambda^{\prime \prime}}$ is the predictive makespan according to the initial schedule that is determined according to the occurred scenario $\lambda^{\prime \prime}$.

\subsubsection{Stability}

It is the degree of rearrangement of jobs (sequence, start-times, and so on) after rescheduling [27]. In this paper, this measure is defined as the difference between the completion times of the jobs in the initial schedule and the realized ones [23]. When a disruption occurs, the real sequence may change after a needed rescheduling. This matter may lead to additional costs, including the costs of reallocation of tools and equipment, reordering raw materials, etc. However, when the real schedule is closer to the initial one, these costs are reduced and stability increases. On the other hand, stability is concerned with the difference between initial and realized schedules themselves rather than between their performances. Therefore, stability measure is defined as absolute deviation in job completion times as follows:

$$
\text { Stability measure : } \sum_{i=1}^{2} \sum_{j=1}^{n} \sum_{r=1}^{n}\left|C R_{i j r}-C P_{i j r}^{\lambda^{\prime \prime}}\right|,
$$

$C R_{i j r}$ is the real completion time of job $j$ on the machine $i$ in position $r$, and $C P_{i j r}^{\lambda^{\prime \prime}}$ is the predictive completion time of job $j$ on machine $i$ in position $r$ under occurred scenario $\lambda^{\prime \prime}$.

\subsubsection{Nervousness}

Schedules are intrinsically nervous and fragile with some unexpected information. This information is not 
known a priori in the planning phase and is revealed over time; thus, dynamic or on-line scheduling techniques are usually used $[27,28]$. To show the influence of nervousness in scheduling problems, another approach is developed in this paper and a new measure, such as the measure of robustness or stability, is defined. This measure includes the effect of frequency of rescheduling in the system after disruptions. When a disruption occurs in a system and the sequence of jobs changes after rescheduling, the change in the sequence causes nervousness in the system. In fact, internal system components, like the operators, will fall into disarray due to change in the sequence and the more this rescheduling frequency, the higher the nervousness of the system will be. Therefore, when the sequence of one or several jobs in the real schedule changes relative to the initial schedule, the system becomes turbulent and chaotic. In this paper, the concepts of instability and nervousness are differentiated. For example, the jobs may be right-shifted after a failure. In this way, due to the change in the completion time of jobs, the stability measure will be a positive number while the nervousness is zero, because the sequence of jobs does not change.

It is assumed that $P O_{i j}^{\lambda^{\prime \prime}}$ is the predictive position of job $j$ on machine $i$ under occurred scenario $\lambda^{\prime \prime}$ and $R O_{i j}$ is the real position of job $j$ on machine $i$ following a failure. A variable is defined as follows:

$$
N_{i j}=1 \text { if } R O_{i j} \neq P O_{i j}^{\lambda^{\prime \prime}}, \text { and } N_{i j}=0 \text { otherwise. }
$$

In fact, if the position of job $j$ in the real schedule is different from the predictive one, then $N_{i j}=1$. Now, variable $T C O$ is defined as the total change in position of jobs following an unexpected failure; therefore, $T C O=\sum_{i=1}^{2} \sum_{j=1}^{n} N_{i j}$. TCO describes nervousness of scheduling.

\subsubsection{NERS value as a selector measure}

The NERS value is a multicomponent measure for the selection of a suitable heuristic to deal with failure of machines. The final definition of NERS value based on the above defined components is as follows:

$$
\begin{aligned}
& \text { Selection measure }=\alpha \text { (Effectiveness) } \\
& +\beta \text { (Robustness measure })+v \text { (Stability measure) } \\
& +\varphi \text { (Nervousness). }
\end{aligned}
$$

The existing coefficients in this measure indicate the importance of each component. These values are determined by the decision makers of the system, such as managers. Thus, in the proposed reactive stage, when a failure occurs, first, the values of these coefficients are determined by the managers of the production system. To determine the weight of each measure, a sensitivity analysis approach can be used, such as the research presented in [27]. They similarly define a comparison metric with four components for comparing deterministic, robust, and online scheduling. They use a sensitivity analysis to determine the weight of each component. Therefore, in a real case, the analyst can consider different amounts for the classical measure and other three components and the manager can choose the better one according to her/his preferences. Moreover, there are many approaches for determining preference weights in multi-criteria decision making, such as the eigenvector method, weighted least-square method, entropy method, and linear programming technique for multidimensional analysis of preference (LINMAP). The readers are referred to Hwang and Yoon [29] for more information. The definition of NERS value is as follows:

$$
\begin{aligned}
& \text { NERS value }_{H}=\alpha\left(\frac{\left(\sum_{j} C R_{2 j n}\right)_{H}}{\max _{H \in \Pi}\left(\sum_{j} C R_{2 j n}\right)_{H}}\right) \\
& +\beta\left(\frac{\left|\sum_{j} C R_{2 j n}-\sum_{j} C P_{2 j n}^{\lambda^{\prime \prime}}\right|_{H}}{\max _{H \in \Pi}\left|\sum_{j} C R_{2 j n}-\sum_{j} C P_{2 j n}^{\lambda^{\prime \prime}}\right|_{H}}\right) \\
& +v\left(\frac{\sum_{i=1}^{2} \sum_{j=1}^{n} \sum_{r=1}^{n}\left|C R_{i j r}-C P_{i j r}^{\lambda^{\prime \prime}}\right|_{H}}{\max _{H \in \Pi} \sum_{i=1}^{2} \sum_{j=1}^{n} \sum_{r=1}^{n}\left|C R_{i j r}-C P_{i j r}^{\lambda^{\prime \prime}}\right|_{H}}\right) \\
& +\varphi\left(\frac{T C O_{H}}{\max _{H \in \Pi} T C O_{H}}\right), \\
& \alpha+\beta+v+\varphi=1 .
\end{aligned}
$$

A set of heuristics can be compared using the NERS value. In fact, after determining the weights, the NERS value is calculated for each heuristic, such as right shifting, regeneration, etc., and the heuristic with the lowest amount of NERS value is selected as a proper reaction to respond to the disruption. The terms in Eq. (25) will be normalized to enable a reasonable comparison between heuristics [27].

\subsection{Solution methods}

There are different heuristics to deal with unexpected disruptions. More common methods in the literature are regenerations and right shifting methods that are explained in the following subsections.

\subsubsection{Right shifting}

One of the simplest reactions, which are commonly implemented in the literature, following the occurrence of a disruption is right shifting. In this approach, when a failure happens, the jobs are processed with the same previous sequence following the repair of failed machine. In this case, the completion time of jobs will be shifted to right within the repair duration 
$\left(d_{f}\right)$. The schedule may be less stable in this method because the real completion time of jobs may change significantly. The advantage of this method is that, due to no change in the sequence, the costs of setup and startup will be less and the system nervousness will be zero. As mentioned earlier, when the manufacturing process begins, we are absolutely certain about which scenario has occurred. Considering this notion, suppose that $\lambda^{\prime \prime} \in \Omega$ is the scenario which has really occurred after the start of the jobs processing. Now, assume that the system has a failure and the scheduled activities are shifted to the right following the failure. Two hypothetical machine failures are shown in Figure 2. Assume that $\mathrm{j} 1, \mathrm{j} 2$, and $\mathrm{j} 3$ are scheduled based on occurred scenario $\lambda^{\prime \prime}$ according to Figure 2(a). Figure 2(b) and (c) are presented machine failures in different times for machine 1 and machine 2 , respectively. Figure 2 shows the steps of right shifting method following the considered failures.

In general, with a repair duration $d_{f}$ and occurred scenario $\lambda^{\prime \prime}$, the value of real completion time of jobs is obtained as follows:

$$
\begin{aligned}
& r_{j}^{\prime}=\left\{r \in n \mid x_{2 j r}=1\right\} ; \quad \forall j \\
& r_{j}^{\prime \prime}=\left\{r \in n \mid x_{1 j r}=1\right\} ; \quad \forall j .
\end{aligned}
$$

If machine 1 fails:

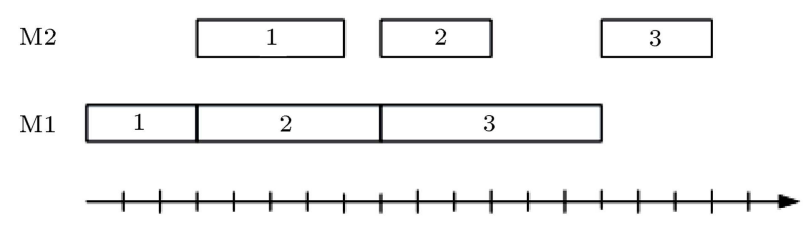

(a)

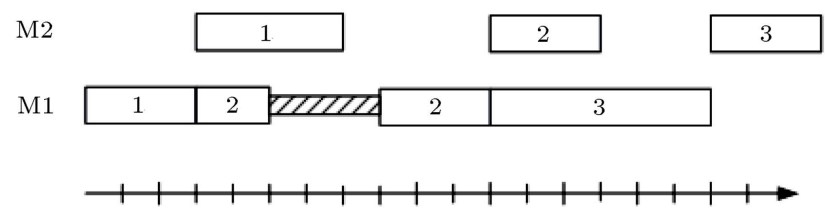

(b)

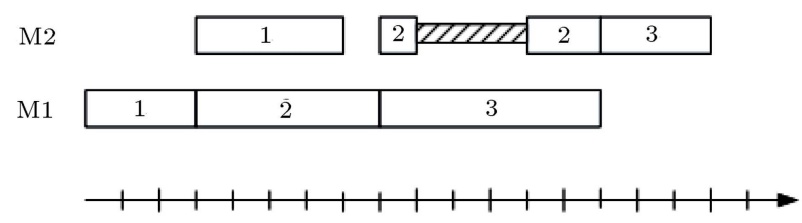

(c)

Figure 2. (a) An initial schedule. (b) Right shifting following a failure on machine 1. (c) Right shifting following a failure on machine 2 .

$$
\begin{aligned}
& C R_{1 j r_{j}^{\prime \prime}}=C P_{1 j r_{j}^{\prime \prime}}^{\lambda^{\prime \prime}}+d_{f} \quad \text { if } C P_{1 j r_{j}^{\prime \prime}}^{\lambda^{\prime \prime}}>t_{f}, \quad \text { and } \\
& C R_{1 j r_{j}^{\prime \prime}}=C P_{1 j r_{j}^{\prime \prime}}^{\lambda^{\prime \prime}}+d_{f} \quad \text { if } \quad C P_{1 j r_{j}^{\prime \prime}}^{\lambda^{\prime \prime}} \leq t_{f} ; \quad \forall j, \\
& C R_{2 j r_{j}^{\prime}}=\max \left\{\sum_{j} C R_{2 j\left(r_{j}^{\prime}-1\right)}, C R_{1 j r_{j}^{\prime \prime}}\right\}+t_{2 j} ;
\end{aligned}
$$

If machine 2 fails:

$$
\begin{aligned}
& C R_{1 j r_{j}^{\prime \prime}}=C P_{1 j r^{\prime} j}^{\lambda^{\prime \prime}} ; \quad \forall j, \\
& S P_{i j r}=C P_{i j r}^{\lambda^{\prime \prime}}-t_{i j} ; \quad \forall i, j, r, \\
& C R_{2 j^{\prime} r_{j^{\prime}}^{\prime}}=C P_{2 j^{\prime} r_{j^{\prime}}^{\prime}}^{\lambda^{\prime \prime}} . \\
& \text { if } \quad j^{\prime}=\left\{j \in J \mid C P_{2 j^{\prime} r_{j^{\prime}}^{\prime}}^{\lambda^{\prime \prime}} \prec t_{f} \text { and } S P_{2 j r_{j}^{\prime}}^{\lambda^{\prime \prime}} \prec t_{f}\right\} \text {; } \\
& \forall j^{\prime} \\
& C R_{2 j^{\prime \prime} r_{j^{\prime \prime}}^{\prime}}=C P_{2 j^{\prime \prime} r_{j^{\prime \prime}}^{\lambda^{\prime \prime}}}^{\prime \prime} \\
& \text { if } \quad j^{\prime \prime}=\left\{j \in J \mid C P_{2 j r_{j}^{\prime}}^{\lambda^{\prime \prime}} \succ t_{f} \text { and } S P_{2 j r_{j}^{\prime}}^{\lambda^{\prime \prime}} \prec t_{f}\right\} \text {; } \\
& \forall j^{\prime \prime}, \\
& C R_{2 j^{\prime \prime \prime} r_{j \prime \prime}^{\prime \prime}}=\max \left\{\sum_{j} C R_{2 j\left(r_{j}^{\prime}-1\right)}, C R_{1 j^{\prime \prime \prime} r_{j^{\prime \prime}}^{\prime \prime}}\right\}+t_{2 j^{\prime \prime \prime}} \\
& \text { if } \quad j^{\prime \prime \prime}=\left\{j \in J \mid C P_{2 j r_{j}^{\prime}}^{\lambda^{\prime \prime}} \succ t_{f} \text { and } S P_{2 j r_{j}^{\prime}}^{\lambda^{\prime}} \succ t_{f}\right\} \text {; } \\
& \forall j^{\prime \prime \prime} \text {. }
\end{aligned}
$$

\subsubsection{Regeneration}

Another method that can be adopted as a rescheduling method following a failure is regeneration heuristic. It reschedules the set of jobs not processed before the rescheduling point and affected by the disruption. In this approach, all jobs that have not yet been processed are completely rescheduled. In this case, the sequence of jobs may change and the system may become stressed and chaotic, but on the other hand, this change of sequence causes good improvement in the effectiveness (objective function).

\subsection{A numerical example}

Consider a two-machine flow shop scheduling problem with five jobs; the processing times of jobs with fewer than three scenarios are given in Table 1. The probabilities for the scenarios are $p^{1}=0.3, p^{2}=0.5$, 
Table 1. Processing time for job $j$ on machine $i$ under scenario $\lambda$.

\begin{tabular}{|c|c|c|c|c|c|c|}
\hline \multirow{2}{*}{ Scenario } & \multirow{2}{*}{ Machine } & \multicolumn{5}{|c|}{$\begin{array}{l}\text { Job processing time } \\
\text { (min) }\end{array}$} \\
\hline & & 1 & 2 & 3 & 4 & 5 \\
\hline \multirow[t]{2}{*}{1} & 1 & 1 & 3 & 7 & 9 & 3 \\
\hline & 2 & 2 & 5 & 7 & 3 & 3 \\
\hline \multirow[t]{2}{*}{2} & 1 & 4 & 2 & 6 & 8 & 5 \\
\hline & 2 & 4 & 6 & 9 & 5 & 2 \\
\hline \multirow[t]{2}{*}{3} & 1 & 2 & 4 & 8 & 7 & 6 \\
\hline & 2 & 3 & 4 & 10 & 2 & 3 \\
\hline
\end{tabular}

and $p^{3}=0.2$, respectively. In the first stage, we should solve this problem based on the proposed model that is developed based on the robust optimization approach. We solve that model with the software GAMS23.6. Based on the outcome of this software, the initial schedule for machines 1 and 2 is " $j 2, j 1, j 3, j 4, j 5$ ". This solution is an initial robust schedule for this problem. We assume that when processing begins, the second scenario will really occur $\left(\lambda^{\prime \prime}=2\right)$.

Now, assume that machine 1 fails at the time point $9\left(t_{f}=9\right)$ and the length of the repair time for it is $d_{f}=3$. We should react properly to this unexpected disruption and reduce its effects based on the proposed multi-criteria measure of "NERS value". We assume that the heuristic methods that we can choose are regeneration and right shifting, so we calculate the NERS value for each of them and select the best. At first, we should determine coefficients of this measure. These parameters are determined as: $\alpha=0.4, \beta=0.2$, $v=0.3$, and $\varphi=0.1$. Table 2 shows the obtained results for both regeneration and right shifting. The results indicate that following this assumptive failure, the regeneration method should be chosen because it has smaller NERS value.

Since the time point of machine failure, the machine that may fail, and $d_{f}$ (length of repair time) are stochastic variables, we extend this problem with simulating failures. We assume that $t_{f}$ is generated from a uniform distribution such as uniform $=[0.001,20]$; also, $d_{f}$ is generated based on uniform $=[5,10]$ and the machine that will fail is chosen stochastically. Then, we consider three levels of the coefficients in the NERS value that are shown in Table 3 . We have run the problem for each level of coefficients at 100 repetitions and, finally, the mean values of effectiveness and performance objectives have been calculated. The results are shown in Table 4 . The worst makespan of
Table 3. Three kinds of test problem for setting the coefficients.

\begin{tabular}{ccccc}
\hline Test problem & \multicolumn{4}{c}{ Coefficient } \\
\cline { 2 - 5 } & $\boldsymbol{\alpha}$ & $\boldsymbol{\beta}$ & $\boldsymbol{\gamma}$ & $\boldsymbol{\varphi}$ \\
\hline 1 & 0.5 & 0.15 & 0.3 & 0.05 \\
2 & 0.4 & 0.2 & 0.3 & 0.1 \\
3 & 0.4 & 0.3 & 0.1 & 0.2 \\
\hline
\end{tabular}

all iterations that occurs in the simulated failures is shown in column 7 and the NERS values are presented in column 8. We call our Proactive-Reactive Method PRM for simplicity. Recall that the classical approach is also used as a benchmark.

Classical approach: The algorithm calculates the initial schedules based on the expected value approach. In fact, the objective function in this approach is minimizing the expected value of all makespans that have been computed for each scenarios $(Z=$ $\left.\operatorname{Min} \sum_{\lambda \in \Omega} p^{\lambda} \sum_{j=1}^{n} C P_{2 j n}^{\lambda}\right)$. Then, when a disruption occurs, two reactions may be made. In the first classical approach, after a disruption, the affected jobs shift to right with the length of the repair time. We call this method CA-Ri. It means that the right shifting method will be used following any disruptions. In the second classical approach, after a disruption, the affected jobs regenerate a new schedule based on the objective function. We call this method CA-Re method.

It should mentioned that there is a logical conflict between stability and robustness of the solution. Because for a more robust solution, it may be necessary to change the sequence of jobs, which can increase stability. To show the conflict between the stability and robustness for this problem, assume that $\varphi=0, \alpha=1$; also, suppose that $v=1-\beta$. Then, for three values of $\beta$, the proposed example is solved. The results are shown in Table 5 and Figure 3. According to the plots,

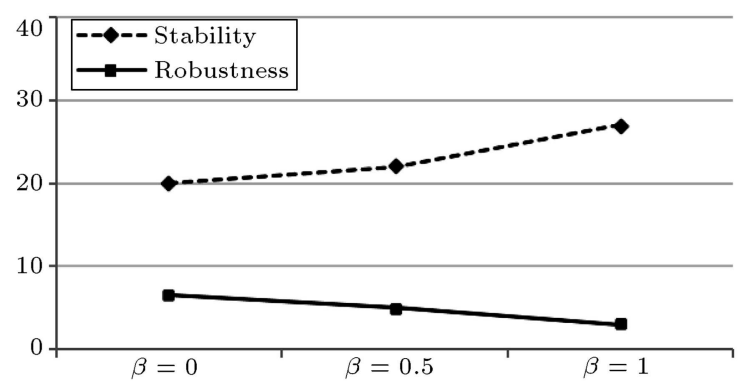

Figure 3. The robustness and stability values.

Table 2. Comparing two heuristics to select following the machine failure.

\begin{tabular}{cccccc}
\hline Approach & Effectiveness & Robustness & Stability & Nervousness & NERS value \\
\hline Regeneration & 31 & 3 & 33 & 5 & 0.581 \\
Right shifting & 41 & 13 & 74 & - & 0.900 \\
\hline
\end{tabular}


Table 4. The mean values of effectiveness and performance objectives according to the accomplished simulations.

\begin{tabular}{cccccccc}
\hline Prob. & Approach & Effectiveness & Robustness & Stability & Nervousness & Worst makespan & NERS value \\
\hline \multirow{2}{*}{1} & PRM & 29.02 & 2.32 & 24.16 & 3 & 30.24 & 0.712 \\
& CA-Ri & 34.97 & 5.77 & 58.01 & - & 36.38 & 0.924 \\
& CA-Re & 28.05 & 2.91 & 65.03 & 2.5 & 32.61 & 0.946 \\
\multirow{2}{*}{2} & & & & & & \\
& PRM & 31.73 & 2.73 & 32.76 & 2 & 35.96 & 0.590 \\
& CA-Ri & 34.61 & 6.11 & 55.87 & - & 33.00 & 0.745 \\
& CA-Re & 31.04 & 3.20 & 85.33 & 3.9 & & \\
\multirow{2}{*}{3} & & & & & & 30.95 & 0.815 \\
& PRM & 31.37 & 2.76 & 32.32 & 2.2 & 37.75 & 0.737 \\
& CA-Ri & 33.99 & 6.09 & 58.18 & - & 35.61 & 0.705 \\
\hline
\end{tabular}

Table 5. The conflict between stability and robustness.

\begin{tabular}{cccc}
\hline Measure & \multicolumn{3}{c}{ Robustness coefficient } \\
\cline { 2 - 4 } & $\boldsymbol{\beta}=\mathbf{0}$ & $\boldsymbol{\beta}=\mathbf{0 . 5}$ & $\boldsymbol{\beta}=\mathbf{1}$ \\
\hline Stability & 20 & 22 & 27 \\
Robustness & 6.6 & 5 & 3 \\
\hline
\end{tabular}

it can be easily deduced that larger value of $\beta$ leads to increase in stability and reduction in robustness.

\section{Experimental design}

Extensive computational experiments are conducted to show the performance of the proposed method. We solved a set of test problems, whose details are given in the following section.

\subsection{Data generation}

- Number of jobs $(n)$ : Seven levels of the number of jobs are considered $(n=5,8,10,12,15,20$, and $25)$. In general, the number of jobs specifies the size of the problem;

- Processing time $\left(t_{i j}^{\lambda}\right)$ : Job processing times are generated from discrete uniform distributions. The unit of the job processing time is minute. The distribution used for $t_{i j}^{\lambda}$ is uniform $\left[a, \eta^{\lambda} b\right]$, where $a=10$ and $b=40$. The parameter $\eta^{\lambda}$ leads to different intervals in processing times for each scenario; it is assumed that $\eta^{1}=1, \eta^{2}=1.5$, and $\eta^{3}=2$;

- Break-down time $\left(t_{f}\right)$ : This parameter explains the time point that a machine fails. The interval between two failures usually follows an exponential distribution with MTBF as the mean time between failures. But, since our proposed method is a reactive one, it is necessary to determine a time point in any simulated run of the problem instead of the interval between failures. Since a machine may fail from the moment zero, the machine begins processing to the moment of proactive makespan, assuming that this parameter is generated from a discrete uniform distribution Uniform $\left[0, C P_{2 j n}^{\lambda^{\prime}}\right]$;
- Duration of repair time $\left(d_{f}\right)$ : This parameter follows an exponential distribution with MTTR as the mean time to repair. Therefore, $d_{f}=e x p-\operatorname{rand}(\mathrm{MTTR})$ and assume MTTR $=20$ time units for any machine.

\subsection{Experimental results}

Seven-type problems defined above are considered based on the number of jobs. In the first stage, each problem is formulated based on the proposed robust model and solved with GAMS23.6 software, and their solutions are determined as the initial robust schedules. Then, machine failures in the scheduling problem are simulated with MATLAB R2007b software and run on a personal computer with a $2.26 \mathrm{GHz}$ processor with 3.00 GB of RAM.

It should be mentioned that our candidate methods to respond to the failures are regeneration and right shifting methods that have been explained in previous sections.

The test problems are simulated in 1000 repetitions and the mean value of each defined criterion is calculated for them. The obtained results for PRM, CA-Ri, and CA-Re are shown in Table 6 and Figure 4.

Table 6 shows details of computational results obtained using PRM, CA-Ri, and CA-Re. These results can be used to evaluate effectiveness and efficiency of the proposed solution methods. A general review of the results in Table 6 and Figure 4 shows that:

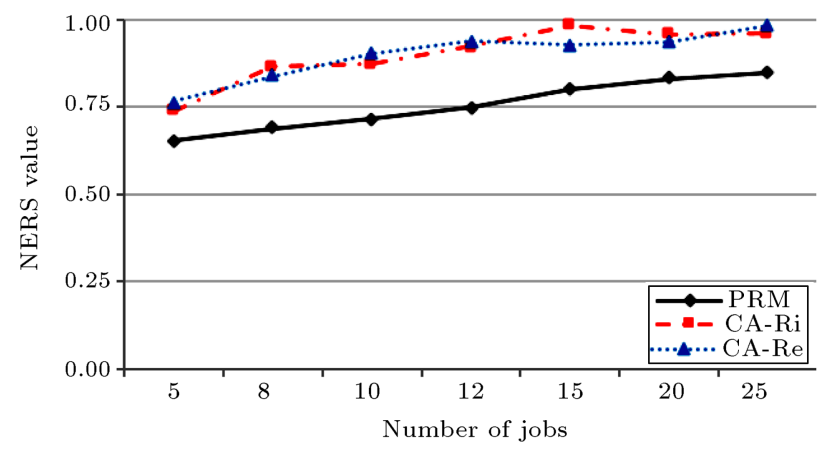

Figure 4. A comparison between NERS values of different solution methods. 
Table 6. The mean values of effectiveness and performance criteria according to solution methods.

\begin{tabular}{|c|c|c|c|c|c|c|c|}
\hline $\begin{array}{c}\text { Number } \\
\text { of jobs }\end{array}$ & Method & Effectiveness & Robustness & Stability & Nervousness & $\begin{array}{c}\text { Worst } \\
\text { makespan }\end{array}$ & $\begin{array}{c}\text { NERS } \\
\text { value }\end{array}$ \\
\hline \multirow{3}{*}{$n=5$} & PRM & 307.14 & 20.14 & 214.22 & 5.2 & 320.42 & 0.6564 \\
\hline & CA-Ri & 290.97 & 51.17 & 415.87 & - & 316.14 & 0.7674 \\
\hline & $\mathrm{CA}-\mathrm{Re}$ & 285.78 & 48.15 & 395.01 & 6.8 & 305.29 & 0.7381 \\
\hline \multirow{3}{*}{$n=8$} & PRM & 324.5 & 29.06 & 320.58 & 7.7 & 346.45 & 0.6895 \\
\hline & CA-Ri & 339.73 & 23.33 & 356.56 & - & 371.45 & 0.8388 \\
\hline & CA-Re & 312.8 & 27.42 & 357.91 & 6.1 & 337.00 & 0.8651 \\
\hline \multirow{3}{*}{$n=10$} & PRM & 375.14 & 32.67 & 379.58 & 11.5 & 404.87 & 0.7162 \\
\hline & $\mathrm{CA}-\mathrm{Ri}$ & 419.02 & 49.14 & 423.13 & - & 505.47 & 0.9017 \\
\hline & CA-Re & 370.66 & 30.76 & 409.76 & 8.4 & 420.12 & 0.8703 \\
\hline \multirow{3}{*}{$n=12$} & PRM & 384.03 & 37.89 & 388.5 & 10.7 & 429.00 & 0.7501 \\
\hline & CA-Ri & 423.91 & 34.02 & 421.09 & - & 450.53 & 0.9382 \\
\hline & CA-Re & 373.76 & 36.87 & 499.32 & 8.3 & 457.91 & 0.9234 \\
\hline \multirow{3}{*}{$n=15$} & PRM & 418.17 & 35.65 & 419.77 & 11.9 & 456.86 & 0.8019 \\
\hline & CA-Ri & 436.90 & 41.60 & 446.81 & - & 470.90 & 0.9278 \\
\hline & $\mathrm{CA}-\mathrm{Re}$ & 421.87 & 47.98 & 497.06 & 13.9 & 450.07 & 0.9631 \\
\hline \multirow{3}{*}{$n=20$} & PRM & 423.40 & 46.33 & 467.50 & 14.0 & 550.32 & 0.8315 \\
\hline & CA-Ri & 512.94 & 57.87 & 469.45 & - & 578.98 & 0.9590 \\
\hline & CA-Re & 456.82 & 52.80 & 517.90 & 16.1 & 498.55 & 0.9351 \\
\hline \multirow{3}{*}{$n=25$} & PRM & 562.06 & 49.65 & 499.77 & 16.5 & 581.06 & 0.8498 \\
\hline & CA-Ri & 587.21 & 65.40 & 476.81 & - & 600.53 & 0.96 .01 \\
\hline & CA-Re & 571.87 & 65.36 & 547.06 & 19.9 & 530.07 & 0.9839 \\
\hline
\end{tabular}

- The NERS value of PRM is lower than the NERS values of the two other methods in each level of jobs. These results show the efficiency of the proposed approach compared to two common classical methods;

- The NERS value for all solution methods will be worse when the problem size increases.

With regard to Table 6 , the effectiveness of PRM is better than that of other methods for $n>=15$. This fact indicates that in addition to NERS, this method also works much better in terms of makespan value of systems with large numbers of jobs.

As mentioned before, the existing coefficients in the NERS value measure indicate the importance level of each component. These values are determined by system decision makers such as managers. Changing the values of these coefficients will cause change in the proper reaction. To consider the impact of these coefficients, we simulated the previous system for 8 different levels of coefficients for two different numbers of jobs ( $n=5$ and $n=8$ ) to analyze of the influence of robustness and stability. The values of each component are reported in Tables 7 and 8 .

The impact of the stability in the NERS value is considered in Table 7 , in which for $n=5$ in rows 2 and $3, \alpha$ and $\varphi$ are fixed, and $\gamma$ and $\beta$ change; then, in rows 4 and $5, \alpha$ and $\beta$ are fixed, and $\gamma$ and $\varphi$ change.

The results show that when the coefficient of stability increases and the coefficient of nervousness decreases while two other coefficients $(\alpha, \beta)$ are fixed, the value of stability decreases because its importance increases in the NERS measure. The NERS values in the last column indicate that the coefficient of stability has a strong influence on NERS measure. The same conclusion can be drawn when the coefficient of stability increases and the coefficient of the robustness decreases while two other coefficients $(\alpha, \varphi)$ are fixed. There are similar conclusions for $n=8$. 
Table 7. Stability analysis based on different coefficients of the NERS value components.

\begin{tabular}{cccccccccc}
\hline $\begin{array}{c}\text { Number } \\
\text { of jobs }\end{array}$ & $\boldsymbol{\alpha}$ & $\boldsymbol{\beta}$ & $\boldsymbol{\gamma}$ & $\boldsymbol{\varphi}$ & Effectiveness & Robustness & Stability & Nervousness & NERS value \\
\hline \multirow{2}{*}{$n=5$} & 0.5 & 0.3 & 0.15 & 0.05 & 294.89 & 26.70 & 161.12 & 3.5 & 0.51 \\
& 0.5 & 0.3 & 0.05 & 0.15 & 306.85 & 18.80 & 219.65 & 3.9 & 0.62 \\
& 0.5 & 0.05 & 015 & 0.3 & 341.90 & 57.32 & 182.29 & 3.3 & 0.66 \\
& 0.5 & 0.15 & 0.05 & 0.3 & 351.82 & 53.56 & 376.01 & 1.7 & 0.73 \\
$n=8$ & & & & & & & & \\
& 0.5 & 0.3 & 0.15 & 0.05 & 412.90 & 72.39 & 229.72 & 5.9 & 0.65 \\
& 0.5 & 0.3 & 0.05 & 0.15 & 355.90 & 53.18 & 210.03 & 8.2 & 0.73 \\
& 0.5 & 0.15 & 0.05 & 0.3 & 391.56 & 80.09 & 420.76 & 3.3 & 0.71 \\
\hline
\end{tabular}

Table 8. Robustness analysis based on different coefficients of the NERS value components.

\begin{tabular}{cccccccccc}
\hline $\begin{array}{c}\text { Number } \\
\text { of jobs }\end{array}$ & $\boldsymbol{\alpha}$ & $\boldsymbol{\beta}$ & $\boldsymbol{\gamma}$ & $\boldsymbol{\varphi}$ & Effectiveness & Robustness & Stability & Nervousness & NERS value \\
\hline \multirow{2}{*}{$n=5$} & 0.5 & 0.15 & 0.3 & 0.05 & 295.89 & 27.73 & 279.17 & 3.7 & 0.56 \\
& 0.5 & 0.05 & 0.3 & 0.15 & 320.99 & 34.57 & 201.98 & 3.2 & 0.61 \\
& 0.5 & 0.2 & 0.1 & 0.2 & 295.89 & 24.70 & 279.17 & 3.6 & 0.63 \\
& 0.5 & 0.1 & 0.2 & 0.2 & 339.31 & 47.23 & 190.03 & 2.1 & 0.69 \\
$n=8$ & & & & & & & & \\
& 0.5 & 0.15 & 0.3 & 0.05 & 393.98 & 81.32 & 296.43 & 2.6 & 0.64 \\
& 0.5 & 0.2 & 0.1 & 0.2 & 421.99 & 80.91 & 309.07 & 4.1 & 0.75 \\
\end{tabular}

Table 8 shows the impact of robustness in the NERS value. The results indicate that robustness has a strong influence on NERS measure, too.

Tables 7 and 8 show that stability is more effective than robustness; thus, a moderate amount is required.

Moreover, the tables demonstrate that when $\alpha$ and $\varphi$ are fixed and $\beta$ and $\gamma$ change, a natural conflict between stability and robustness occurs. Based on the results, when $\beta$ increases and $\gamma$ decreases, stability and robustness change conversely and this shows the conflict between these two measures.

Furthermore, the NERS values for these different levels of coefficients are considered to gain a proper tradeoff between these different components and decision makers can choose the best ones based on their opinions.

\subsubsection{Comparing effectiveness of solution methods}

The NERS measure is used to evaluate the performance of the solution methods and to show effectiveness of the proposed method. A single factor ANOVA is used to find out whether there is a significant difference among the performances of solution methods. Data have been made normal and they have equal variances (tested by MINITAB14 software). ANOVA results are presented in Table 9 . These results with $P$-value $=$ 0.003 confirm that there is at least one method with different mean response when confidence level is set at 0.95. Thus, Fisher's least significant difference method is used to compare the performances of methods (see Table 10). The results confirm that there is a significant difference between PRM, CA-Ri, and CA-Re. Also, Table 9 shows no significant difference between CA-Ri and CA-Re.

As Figure 5 illustrates, among the solution methods, proactive-reactive method for NERS value is better than both the classical approach with right shifting and the classical approach with regeneration.

Table 9. ANOVA results for solution methods.

\begin{tabular}{cccccc}
\hline Source & df & SS & MS & F & $\boldsymbol{P}$-value \\
\hline Algorithm & 2 & 0.09347 & 0.04674 & 8.07 & 0.003 \\
Error & 18 & 0.10426 & 0.00579 & & \\
Total & 20 & 0.19773 & & & \\
\hline
\end{tabular}


Table 10. Fisher $95 \%$ individual confidence intervals for all pair-wise comparisons.

\begin{tabular}{ccccc}
\hline Algorithms & Lower & Center & Upper & $\begin{array}{c}\text { Significant difference } \\
\text { at 95\% level }\end{array}$ \\
\hline PRM vs. CA-Ri & 0.057 & 0.143 & 0.228 & Yes \\
PRM vs. CA-Re & 0.055 & 0.141 & 0.226 & Yes \\
CA-Ri vs. CA-Re & -0.087 & -0.002 & 0.083 & No \\
\hline
\end{tabular}

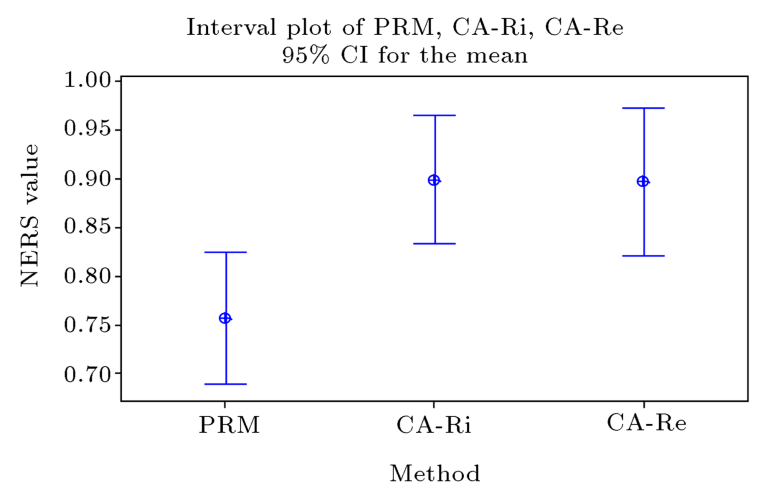

Figure 5. Means and interval plot for NERS value.

Therefore, the superiority of the proposed solution approach is concluded over other heuristics including $\mathrm{CA}-\mathrm{Ri}$ and $\mathrm{CA}-\mathrm{Re}$ due to the computational results.

\section{Conclusions}

In this paper, a proactive-reactive approach was presented instead of SMs. This two-stage approach produced robust and stable solutions for a two-machine scheduling problem in a flow shop environment. In this approach, firstly, by considering uncertain processing times and using the robust optimization approach, the problem was solved and a robust initial solution was proactively produced. Then, in case of machine failure, the appropriate reaction was adopted based on the defined performance measure. This measure was a multi-criteria measure defined in terms of solution effectiveness, robustness, stability, and reduction of system nervousness. Computational results indicated that this method was much more effective than the ordinary scheduling methods that operated on the basis of makespan alone. The results showed efficiency of the proposed approach compared to two common classical methods in the face of unexpected failures. For future research, this problem can be considered for other scheduling problems of shop floor, and other classical objectives can be used to evaluate this method. As another research subject, random distributions can be considered for the occurrence time of failure and repair duration, because they can be used for obtaining initial solutions that are proactively able to reduce the effect of machine breakdown. Moreover, the effect of this approach on other random disruptions such as the arrival of new jobs, order cancellations, etc. can be investigated and analyzed. Another important future research could be the presentation of proper heuristic methods for machine failure, which can be applied in the second step of this approach.

\section{References}

1. Beck, J.C. "Solution-guided multi-point constructive search for job shop scheduling", J. Artif. Intell. Res., 29, pp. 49-77 (2007).

2. Beck, J.C. and Wilson, N. "Proactive algorithms for job shop scheduling with probabilistic durations", $J$. Artif. Intell. Res., 28, pp. 183-232 (2007).

3. Sabuncuoglu, I. and Goren, S. "Hedging production schedules against uncertainty in manufacturing environment with a review of robustness and stability research", Int. J. Comput. Integr. Manuf., 22(2), pp. 138-157 (2009).

4. Ouelhadj, D. and Petrovic, S. "A survey of dynamic scheduling in manufacturing systems", J. Scheduling., 12, pp. 417-43 (2009).

5. Kuchta, D. "A concept of a robust solution of a multi criteria linear programming problem", Cent. Eur. J. Oper. Res., 19, pp. 605-613 (2011).

6. O'Donovan, R., Uzsoy, R. and McKay, K.N. "Predictable scheduling of a single machine with breakdowns and sensitive jobs", Int. J. Prod. Res., 37(18), pp. 4217-4233 (1999).

7. Pinedo, M.L., Scheduling: Theory, Algorithms, and Systems, Springer, 3rd Edition (2008).

8. Fattahi, P., Hosseini S.M.H. and Jolai, F. "A mathematical model and extension algorithm for assembly flexible flow shop scheduling problem", Int. J. Adv. Manuf., 65(5-8), pp. 787-802 (2012).

9. Daniels, R. and Kouvelis, P. "Robust scheduling to hedge against processing time uncertainty in single stage production", Manag. Sci., 41(2), pp. 363-376 (1995).

10. Kouvelis, P., Kurawarwala, A.A. and Gutierrez, G.J. "Algorithms for robust single- and multiple-period layout planning for manufacturing systems", Eur. J. Oper. Res., 63, pp. 287-303 (1992).

11. Mulvey, J.M., Vanderbei, R.J. and Zenios, S.A. "Robust optimization of large-scale systems", Oper. Res., 43, pp. 264-281 (1995).

12. Rossi, A. "A robustness measure of the configuration of multi-purpose machines", Int. J. Prod. Res., 48(4), pp. 1013-1033 (2010). 
13. Kutanoglu, E. and Sabuncuoglu, I. "Experimental investigation of iterative simulation-based scheduling in a dynamic and stochastic job shop", J. Manuf. Syst., 20(4), pp. 264-279 (2001).

14. Wu, S.D., Storer, R.N. and Chang, P. "One-machine rescheduling heuristics with efficiency and stability as criteria", Comput. Oper. Res., 20(1), pp. 1-14 (1993).

15. Leon, V.J., Wu, S.D. and Storer, R.H. "Robustness measures and robust scheduling for job shops", IIE. Trans., 26(5), pp. 32-43 (1994).

16. Lawrence, S.R. and Sewell, E.C. "Heuristic, optimal, static, and dynamic schedules when processing times are uncertain", J. Oper. Manag., 15, pp. 71-82 (1997).

17. Sabuncuoglu, I. and Karabuk, S. "Rescheduling frequency in an FMS with uncertain processing times and unreliable machines", J. Manufacturing., 18(4), pp. 268-283 (1999).

18. Jensen, M.T. "Generating robust and flexible job shop schedules using genetic algorithms", IEEE. Trans. Evolut. Comput., 7(3), pp. 275-288 (2003).

19. Goren, S. and Sabuncuoglu, I. "Robustness and stability measures for scheduling: single machine environment", IIE. Trans., 40(1), pp. 66-83 (2008).

20. Sotskov, Y.N., Egorova, N.G. and Lai, T.C. "Minimizing total weighted flow time of a set of jobs with interval processing times", Math. Comput. Model., 50, pp. 556-73 (2009).

21. Bouyahia, Z., Bellalouna, M., Jaillet, P. and Ghedira, K. "A priori parallel machines scheduling", Comput. Ind. Eng., 58(3), pp. 488-500 (2010).

22. Ghezail, F., Pierreval, H. and Hajri-Gabouj, S. "Analysis of robustness in proactive scheduling: A graphical approach", Comput. Oper. Res., 58, pp. 193-198 (2010).

23. Al-Hinai, N. and ElMekkawy, T.Y. "Robust and stable flexible job shop scheduling with random machine breakdowns using a hybrid genetic algorithm", Int. J. Prod. Econ., 132, pp. 279-291 (2011).

24. Al-Hinai, N. and ElMekkawy, T.Y. "An efficient hybridized genetic algorithm architecture for the flexible job shop scheduling problem", Flex. Serv. Manuf. J., 23, pp. 64-85 (2011).

25. Leung, S.C.H. and Wu, Y. "A robust optimization model for stochastic aggregate production planning", Prod. Plan. Control., 15(5), pp. 502-514 (2004).

26. YU, C.S. and LI, H.L. "A robust optimization model for stochastic logistic problems", Int. J. Prod. Econ., 64, pp. 385-397 (2000).

27. Gan, H.S. and Wirth, A. "Comparing deterministic, robust and online scheduling using entropy", Int. J. Prod. Res., 43(10), pp. 2113-2134 (2005).

28. Rahmani, D. and Heydari, M. "Robust and stable flow shop scheduling with unexpected arrivals of new jobs and uncertain processing times", J. Manuf. Syst., 33(1), pp. 84-92 (2014).

29. Hwang, C.L. and Yoon, K., Multiple Attribute Decision Making: Methods and Applications, Springer-Verlag, Berlin (1981).

\section{Biography}

Donya Rahmani is an Assistant Professor of Industrial Engineering at K. N. Toosi University of Technology. She received her BSc degree in Industrial Engineering from Bu-Ali Sina University and MSc and $\mathrm{PhD}$ degrees both in Industrial Engineering from Iran University of Science and Technology. Her research interests are in production planning, robust optimization, dynamic scheduling, and operations research. She is a reviewer of several international journals. 